This is the accepted manuscript of:

L. Pantani, A. Brioli, P. Tacchetti, B.A. Zannetti, K. Mancuso, S. Rocchi, M. Martello, I. Rizzello, C. Terragna, E. Zamagni \& M. Cavo (2016) Current and emerging triplet combination therapies for relapsed and refractory multiple myeloma, Expert Review of Hematology, 9:3, 315-323, DOI:

Available at https://doi.org/10.1586/17474086.2016.1127754

Rights / License:

The terms and conditions for the reuse of this version of the manuscript are specified in the publishing policy. For all terms of use and more information see the publisher's website.

This item was downloaded from IRIS Università di Bologna (https://cris.unibo.it/)

When citing, please refer to the published version. 


\section{Current and emerging triplet combination therapies for relapsed and refractory multiple}

myeloma

Pantani L.* , Brioli A.*, Tacchetti P., Zannetti B.A., Mancuso K., Rocchi S., Martello M., Rizzello I., Terragna C., Zamagni E., Cavo M.

Affiliation: "Seràgnoli" Institute of Hematology, Bologna University School of Medicine, Bologna, Italy

*These authors contributed equally to this work

Correspondence:

Elena Zamagni MD

Istituto di Ematologia ed Oncologia Medica "Seràgnoli"

Dipartimento di Medicina Specialistica, Diagnostica e Sperimentale

Università degli Studi di Bologna

Policlinico S. Orsola-Malpighi

Via Massarenti, 9 - 40138 Bologna, Italy

Phone +390516363680

Fax +390516364037

e-mail: e.zamagni@unibo.it 


\section{Summary}

Despite significant improvement in outcomes have been observed for multiple myeloma (MM) patients over the past 10-15 years, mainly due to the introduction of novel agents targeting the tumor clone and the bone marrow microenvironment, treatment of refractory and/or relapsed (RR) disease remains a challenge, particularly for patients who have failed prior bortezomib- and lenalidomide-based therapies. More recently, new drugs with different mechanisms of action, including second generation proteasome inhibitors, third generation immunomodulatory drugs, histone deacetylase inhibitors and monoclonal antibodies, have been developed and are under investigation, further increasing treatment options for RRMM patients. Overall, novel agent-based triplet combinations demonstrated superior response rates and prolonged disease control when compared with two-drug regimens in several randomized clinical trials, without adding any relevant additional toxicity. Salvage triplet therapies are likely to play a key role in overcoming drug-resistance and hold promise to further improve long-term outcomes of RRMM patients.

Key Words: multiple myeloma , IMiDs , proteasome inhibitors , monoclonal antibodies , relapsed refractory 


\section{Introduction}

Over the past decade, the introduction and widespread use of novel agents, such as the immunomodulatory drugs (IMiDs) thalidomide and lenalidomide, and the first-in-class proteasome inhibitor (PI) bortezomib, has dramatically improved the prognosis of multiple myeloma (MM) patients, resulting in a significant increase in the depth and duration of responses which have ultimately led to extended survival. [1] These novel drugs represent the backbone of many current standard-of-care therapies for young and elderly patients, both at diagnosis and in the relapse setting.

Despite these major advances, MM remains an incurable malignancy with the majority of patients experiencing relapse and requiring additional therapy. At every subsequent relapse the duration of response is progressively shorter and drug-resistance ultimately emerges. The outcomes of patients refractory to prior bortezomib- and lenalidomide-based therapies are dismal, with median progression-free survival (PFS) of only 5 months and overall survival (OS) of 9 months. [2] Therefore, newer drugs and novel combinations that show promise to overcome resistance to standard therapies are needed for relapsed/refractory (RR) MM.

New insights into the biology of the disease have prompted an intense research focused to the development of additional novel agents targeting specific pathways involved in tumour cell growth and survival. A plethora of new compounds have been extensively investigated in the last few years, both in the preclinical setting and in clinical trials. Due to their effectiveness some of these agents have received fast-track approval and have now moved to phase III and IV studies. In this regard, the third generation IMiD pomalidomide in combination with dexamethasone and the second generation PI carfilzomib as single agent have been approved by U.S. Food and Drug Administration (FDA) in 2013 for the treatment of RRMM patients who have received at least two prior treatment regimens, including both lenalidomide and bortezomib, and have demonstrated 
disease progression on the last therapy. $[3,4]$ Both molecules demonstrated a more potent in vitro activity than their respective first-in class compounds bortezomib and thalidomide. [4-8]

Pomalidomide, a derivate of thalidomide with a modified chemical structure, was demonstrated in vitro to have multiple mechanisms of action including direct antiproliferative, pro-apoptotic, and antiangiogenic effects, and inhibition of stromal cell support. Furthermore, pomalidomide showed modulatory effects on bone resorption and on the immune system. [9] Carfilzomib is an epoxyketone PI that irreversibly and selectively inhibits the chymotripsin-like activity of the $20 \mathrm{~S}$ proteasome, necessitating de novo protein synthesis to restore proteasome's activity. In preclinical studies carfilzomib demonstrates more potent proteasome inhibition with a minimal off-target activity compared to its predecessor bortezomib. In particular, the significantly less cross-reactivity with non-proteasomal proteases has been shown to correlate with a lack of carfilzomib-related neurotoxicity in preclinical studies. $[5,10]$

Although second generation PIs and third generation IMiDs have proven to be effective in the treatment of RRMM, [3,4] other agents with different mechanisms of action are being developed and hold promise to represent promising therapeutic options for MM treatment. Among emerging novel drugs with different mechanisms of action it is worth highlighting monoclonal antibodies (MoAb) and histone deacetylase inhibitors (HDACi).

MoAb are directed against cell-surface receptors that are uniformly expressed by plasma cells and that impact fundamental cellular capabilities such as homing, adhesion and migration. Direct target-binding activates potent cytotoxic immune effector functions such as Ab-dependent cellular cytotoxicity (ADCC), complement-dependent cytotoxicity $(C D C)$, activation of natural killer (NK) cells apoptosis, leading to MM cells lysis. Among the principal MoAb tested in RRMM, elotuzumab, targeting cell surface glycoprotein SLAMF7, failed to induce responses when used as a single agent, whereas significant activity was observed when it was combined either with 
bortezomib or lenalidomide in phase I and II dose-escalation studies. [11,12] On the other hand, the anti-CD38 MoAb daratumumab exerted remarkable antitumor activity, which was further enhanced by the combination with lenalidomide. $[13,14]$

HDACi are involved in the unfolded protein response pathways and in the epigenetic regulation of gene expression, promoting cell proliferation and cell death. Despite demonstration of promising preclinical activity, monotherapy with the pan-HDACi vorinostat failed to provide any clinical benefit, while its use in combination with bortezomib resulted in a relevant increase in toxicity. [15] More promising results have been reported with the pan-HDACi panobinostat that has been granted fast track approval by the FDA in combination with bortezomib and dexamethasone for the treatment of RRMM, based on the increased PFS compared to bortezomib and dexamethasone demonstrated in a phase III study. [16]

\section{Triplet versus double combinations}

From what said it appears evident that various treatment options and drug combinations are currently available for RRMM patients. A growing body of evidence suggests that a three drugbased salvage therapy, often including a novel agent, could represent a key strategy to improve long-term treatment outcomes and overcome drug-resistance compared to standard treatments.

This review provides a summary of the main studies reported so far and aimed at exploring the role of three drug novel agent-based therapies for RRMM patients, with a particular focus on phase III trials prospectively comparing triplet versus doublet regimes. (Table.1) 


\section{First and second generation IMiDs: thalidomide- and lenalidomide-based regimens}

Thalidomide was the first novel drug with well established activity in the RRMM setting, yielding an overall response rate (ORR) of $25 \%$ when used as a single agent, $[17,18]$ and up to $50 \%$ when in combination with high dose dexamethasone. [19]

A higher efficacy was demonstrated with the subsequent addition of chemotherapeutic drugs, such as an alkylator [20,21] or an antracycline [22]. Offidani et al. performed a pair-matched comparison of 94 RRMM patients who received a minimum of 3 to 6 cycles of either thalidomidedexamethasone or the same doublet combined with liposomal pegylated doxorubicin (ThaDD), followed by maintenance therapy with thal-dex until relapse or toxicity. With the limits of a retrospective case-match analysis, ThaDD resulted in a significantly higher rate of at least minimal response ( $92 \%$ vs $63.5 \%$ in the control group, $p<0.001$ ), including a superior rate of $\geq$ partial response (PR, $75.5 \%$ vs $59.5 \%$ ) and $\geq$ near complere response ( $\mathrm{nCR}, 30 \%$ vs $10.5 \%, p=0.02$ ). This gain translated into a prolonged median PFS (21 vs 11.5 months for the control group) and OS (35.5 vs 20 months, respectively) for those patients receiving the triplet combination. [22] As expected the addition of an anthracycline was associated with a higher frequency of hematologic toxicity and infections which, however, were easily managed with adequate prophylaxis and supportive care and did not led to a higher treatment discontinuation. [22]

Results of a phase III randomized trial aimed at comparing the efficacy and safety of the triple combination bortezomib-thalidomide-dexamethasone (VTD) vs thalidomide-dexamathasone (TD) as first salvage therapy in $\mathrm{MM}$ patients relapsing after an autologous stem-cell transplantation were recently reported. [23] All patients received thalidomide at $200 \mathrm{mg}$ daily for 1 year and dexamethasone at the total dose of $160 \mathrm{mg}$ every 3 weeks. In addition, patients allocated to VTD treatment received intravenous bortezomib twice-weekly for the first eight 21day cycles, and then once a week for additional 6 months. As already seen in preclinical studies 
and in the upfront setting [24-26], the addition of bortezomib to TD resulted in superior efficacy in terms of high quality responses and prolonged disease control. On an intention-to-treat basis, VTD yielded a significantly higher rate of at least very good partial response (VGPR) as compared to TD ( $56 \%$ vs $35 \%$, respectively, $p=0.001$ ) and a two-fold higher probability to achieve $C R+n C R$ ( $45 \%$ vs $21 \%, p<0.001)$. Median time to progression was longer in patients receiving VTD than TD, $(19.5$ vs 13.8 months, $p=0.001)$, as well as median PFS (18.3 vs 13.6 months, $p=0.001)$, whereas the 2 year projected OS rates were not significantly different (71\% vs $65 \%)$ between the two groups. Overall, grade 3-4 drug-related adverse events were more frequent in the VTD arm compared to TD ( $59 \%$ vs $36 \%, p<0.001)$. In particular, the most frequent grade $\geq 3$ adverse event was cumulative, dose-related, peripheral neuropathy, which occurred in $29 \%$ of patients in the VTD arm as compared to $12 \%$ in TD $(p=0.001)$. [23]

Lenalidomide in combination with high-dose dexamethasone (RD) was approved for use in RRMM in 2006 on the basis of two parallel randomised phase III trials demonstrating its superiority versus high-dose dexamethasone alone in terms of ORR and median PFS. $[27,28]$ Based on the results of a subsequent study for newly diagnosed MM patients providing demonstration of the inferior short-term OS and higher toxicity of RD compared to lenalidomide and low-dose dexamethasone (Rd), [29] this latter combination has formed the backbone of many triplet therapies for RRMM patients incorporating either a PI or a MoAb, which will be discussed more in details in the following subsections.

\section{Third generation IMiDs: pomalidomide-based regimens}

Pomalidomide combined with low-dose dexamethasone (pom-dex) proved to be a feasible and effective salvage therapy for heavily pre-treated RRMM patients who had exhausted prior responsiveness to bortezomib and lenalidomide. [30,31] In particular, in the MM-03 trial 
comparing pom-dex vs high-dose dexamethasone in patients who had received a median of 5 lines of prior therapy, the doublet pom-dex regimen yielded a 32\% ORR, with median PFS and OS values of 4 and 13 months, respectively. [3]

More recently, the addition of a third drug to pom-dex has been explored in several phase I/II studies, in order to further improving clinical outcomes. Larocca et al. evaluated dosing, safety profile and efficacy of pomalidomide-cyclophosphamide-prednisone (PCP) in patients who had failed 1 to 3 prior lines of therapy, including lenalidomide. Despite using a monthly dose of pomalidomide of $70 \mathrm{mg}$ (inferior to the standard dose of $84 \mathrm{mg} / \mathrm{month}$ ) and a low dose of cyclophosphamide (namely $50 \mathrm{mg}$ every other day), the PCP regimen induced an ORR of $51 \%$ and a median PFS of 10.4 months, with $69 \%$ of patients alive at 1 year. Most common grade $3-4$ hematologic toxicities were consistent with what observed in previous studies. [32]

Similarly, preliminary data from a phase II randomised trial showed that pom-dex combined with oral cyclophosphamide yielded higher ORR and a two-fold longer median PFS (9.2 vs 4.4 months) compared to pom-dex alone. [33]

Even more promising results seemed to emerge for the combination of pom-dex with a first or second generation PI. Impressive rates of at least PR, averaging approximately from 75 to $80 \%$, were reported for both pom-dex-bortezomib and pom-dex-carfilzomib triplets, with median PFS of 12 and 17 months, respectively. [34,35] The combination of pomalidomide-carfilzomibdexamethasone (CPD) is currently being further tested in three ongoing phase I/II clinical trials for RRMM patients previously exposed and refractory or intolerant to lenalidomide. Results from a phase I study including 32 patients previously treated with a median of 6 lines of therapy have recently been published. [36] By study design, all patients were lenalidomide refractory and 91\% were also bortezomib refractory. In this heavily pretreated population, at least a minimal response (MR) was observed in $66 \%$ of the patients, including $16 \%$ of VGPR. The efficacy of the CPD regimen 
was retained across all cytogenetic subgroups, including those high-risk. Side effects were manageable, with the main toxicity being hematological. In particular, $44 \%$ of patients experienced grade III-IV neutropenia, a value superimposable to that seen in this setting using pom-dex alone. Grade III non hematological adverse events occurred in 1 patients each and included congestive heart failure, transient ischemic attack and pulmonary embolus; two treatment related deaths were reported. [36]

\section{Proteasome inhibitors-based regimens}

Based on the results of the phase III APEX trial, the first-in-class PI bortezomib received FDA and EMEA approval in 2005 as single agent therapy for relapsed MM patients who had received al least one prior line of therapy. [37,38] In subsequent phase II studies, the combination of bortezomib and dexamethasone (VD) demonstrated added clinical benefit, without adversely affecting the safety profile, and is actually considered as a reference regimen in the relapse setting. [39]

Based on preclinical demonstration that bortezomib enhanced the sensitivity of myeloma cells to conventional chemotherapy [40] and of its sinergystic clinical activity when combined with other agents, [41] several phase I/II trials have explored the combination of bortezomib with anthracyclines, [42-44], IMiDs [45] and bendamustine [46-48]. All these studies showed that the combination of VD with a third agent in patients exposed to a median of 1 to 5 prior therapies provided a rate of at least PR in the $61-72 \%$ range, with a manageable toxicity. Offidani and colleagues investigated the role of the triplet therapy bendamustine-bortezomib-dexamethasone (BBD) in 75 RRMM patients that had received less than 4 prior lines of therapy. In the 70 evaluable patients treatment with BBD provided an ORR (at least a PR) of $71.5 \%$, with $16 \%$ and $18.5 \%$ of patients achieving a CR and a VGPR, respectively. With a follow-up of 1 year the median time-to- 
progression (TTP) was 16.5 months and the 1-year overall survival was 78\%. [46] Similar results were obtained by the French group, which investigated the BBD regimen in 73 RRMM patients. After 4 cycles of BBD at least a PR was achieved in $57.6 \%$ of patients, with $10.9 \%$ and $16.5 \%$ achieving a CR and a VGPR respectively. With a median follow up of 15.7 months the median PFS was 10.8 months and the median OS was 23 months. [48]

More recently, in the phase III randomized PANORAMA-1 study the combination of VD with the HDCAi panobinostat was compared with VD and placebo in a population of more than 760 RRMM patients. The simultaneous targeting of different pathways involved in the unfolded protein response, e.g. the ubiquitin-proteasome pathway by bortezomib and the aggresome protein degradation pathway by $\mathrm{HDACi}$, provided the rationale for this triplet regimen. The addition of panobinostat to VD resulted in a significantly higher frequency of $C R / n C R(27.6 \%)$ compared to VD-placebo $(15.7 \%)(p<0.0001)$, a gain which translated into a significantly longer PFS (12 vs 8.1 months, $p<0.0001)$. Importantly, PFS benefit was observed across pre-specified subgroups, including patients aged 65 years or older, those with ISS 2-3 and patients previously exposed to bortezomib. At the time of the analysis, no significant difference was reported in median OS (33.6 months for panobinostat and 30.4 for placebo group). The triplet regimen was associated with a superior frequency of grade 3-4 adverse events, including diarrhoea ( $26 \%$ vs $8 \%$ for VD alone), fatigue ( $24 \%$ vs $12 \%)$, and haematological toxicities, mainly thrombocytopenia (67\% vs $31 \%$ ) and lymphopenia (53\% vs $40 \%)$. [16]

As stated above, the combination of lenalidomide with emerging new molecules has been extensively investigated in the last few years. Recently, the ASPIRE trial prospectively compared Rd plus carfilzomib versus Rd in a large population of relapsed myeloma patients who had received 1 to 3 prior treatments. [49] The addition of carfilzomib to Rd for eighteen 28 -day cycles followed by $\mathrm{Rd}$ until progression or undue toxicity resulted in a remarkable improvement of PFS (26.3 months 
vs 17.6 in the control arm, $\mathrm{p}<0.001$ ) and a $31 \%$ decrease in the relative risk of progression or death compared to standard Rd. The advantage in PFS offered by the triplet combination was retained across all predefined subgroups, including patients previously exposed to bortezomib and IMiDs, and those with a high-risk cytogenetic profile at study entry. Two-year estimates of OS were $73.3 \%$ and $65 \%$, respectively $(p=0.04)$. The 3 -drug regimen was associated with a higher rate of CR or better $(31.8 \%$ vs $9.3 \%, p<0.001)$ and a superior ORR $(87 \%$ vs $66.7 \%, p<0.001)$. Overall, no relevant differences were observed between the 2 groups in the frequency of reported grade $\geq$ 3 adverse events or treatment discontinuation due to toxicity, although patients in the carfilzomib arm experienced more common side effects than those treated with Rd, including diarrhea, cough and hypertension. Of note, the incidence of any-grade peripheral neuropathy was $17 \%$ in both groups of patients. [49] Based on these results, in July 2015 the FDA approved Carfilzomib in combination with lenalidomide and dexamethasone for the treatment of patients with relapsed MM who have received 1 to 3 prior lines of therapy.

Based on its activity as single agent in RRMM patients previously exposed to a median of 4 prior lines of treatment, [50] the oral PI ixazomib is also being evaluated in combination with Rd versus the doublet Rd in an ongoing phase III clinical trial.

\section{Monoclonal antibodies-based regimens}

Among the new agents explored for the treatment of MM, MoAbs have emerged as an attractive targeted strategy based on the wide range of antigens expressed on the surface of plasma cells, in analogy with what previously seen in non Hodgkin Lymphoma, where the advent of the antiCD20 MoAbs dramatically changed the treatment scenario. [51,52] Encouraging results have been recently provided by the ELOQUENT-2 trial, a phase III randomised study comparing the efficacy and safety of Rd with or without elotuzumab in RRMM patients. [53] The rationale for 
combining a MoAb with an IMiD is based on the synergistic effect of direct activation and engagement of the host's natural killer cells by elotuzumab, and the enhancement of antibodydependent cellular toxicity exerted by lenalidomide. Six hundred forty-six patients, with 1 to 3 prior therapies and a documented disease progression after their most recent treatment, were randomly assigned to receive $\mathrm{Rd}$ alone until disease progression or unacceptable toxicity, or the same combination plus elotuzumab. The combination Elotuzumab-Rd resulted in an extended PFS as compared with the control arm (19.4 months vs 14.9 months, respectively, $p<0.001)$, a benefit maintained regardless of patients age, number of prior therapies, previous exposure to lenalidomide or the presence of high-risk cytogenetic abnormalities, such as del $17(p)$ or $t(4 ; 14)$. The ORR was $79 \%$ in the elotuzumab group vs $66 \%$ in the control group $(p<0.001)$. Concerning safety profile, the most common grade 3-4 adverse events were lymphocytopenia, which was reported in $77 \%$ of patients receiving elotuzumab and $49 \%$ of patients in the $\mathrm{Rd}$ arm, neutropenia and fatigue. Infusion reactions to elotuzumab occurred in $10 \%$ of patients and were of mild grade. [53]

In another recent phase II study, 152 patients were randomized to receive either VD alone or VD in combination with Elotuzumab until disease progression or unacceptable toxicity. Despite the achievement of a similar ORR, the triplet regimen improved median PFS (9.7 vs 6.9 months for $V D, p=0.08)$. More common $\geq$ grade 3 adverse events were thrombocytopenia and infections, with mild grade 1-2 infusion reactions occurring in 7\% of patients in the elotuzumab group. [54] Based on the favourable toxicity profile and efficacy of the anti-CD38 MoAb Daratumumab as single agent in heavily pretreated and refractory MM patients, [55] subsequent phase I/II studies explored Daratumumab combined with Rd in RRMM, with a further enhanced ORR, averaging 75\%. $[14,56,57]$ These results prompted the initiation of phase III clinical trials of Daratumumab in combination with either Rd or VD, whose results are eagerly awaited. The combination of 
daratumumab with pomalidomide and dexamethasone is also being evaluated in ongoing phase I trials. [58]

\section{Expert commentary}

The plethora of emerging novel drugs currently under investigation and in various stages of clinical development has dramatically expanded the therapeutic armamentarium for RRMM. However, in the absence of prospective randomized trials directly comparing the different newer combinations in the same setting of patients, a standard of care for the management of RRMM cannot be defined. More conventional regimens, such as those including alkylators and conventionally used in newly diagnosed patients should also not be forgotten, as they can still have a role in the RR setting. In this respect regimens such as Bortezomib-Melphalan-Prednisone (VMP) or Bortezomib-Cyclophosphamide-Dexamethasone (VCD), or even the more old combinations MP and $\mathrm{CP}$, could be considered for the treatment of RRMM patients, proven that patients are not refractory to these drugs.

With so many available treatment options, and in the lack of a defined standard of care for this category of patients, the choice of optimal salvage therapy and treatment sequencing still remains a special challenge and depends on several disease-related and patient-related factors, such as risk stratification, age, comorbidities, previous therapies actually received and related quality and duration of response, not to mention drug availability and costs. In addition, a careful balance between efficacy and potential side effects is required, in particular considering that patients with advanced or end-stage $\mathrm{MM}$ are more fragile and at risk of developing severe complications compared with those at earlier relapse. 


\section{Five years view}

Despite the caveat mentioned above (possible higher toxicities for 3-drugs compared to 2-drugs regimen), a three-drug based therapy for RRMM is likely to be superior to a double regimen. This considerations are based on the results of several large, phase III clinical trials, demonstrating that the addiction of a third drug to the backbone of Rd and VD increases the depth and duration of response and prolongs PFS up to unprecedented values, sometimes rivalling those seen in newly diagnosed disease, without meaningfully affecting patient safety and quality of life. Thanks to these trials most of this newer drugs have received, or are currently receiving, FDA and EMA approval, making it possible also for patients outside clinical trials to benefit from them. In light of these observations, triplet combination regimens actually represent a key option in overcoming drug resistance and improving long-term treatment outcomes in RRMM patients. The availability of multiple drugs with different mechanism of actions makes it reasonable to postulate that in the future a plethora of therapeutic regimens, differently combining PI, IMiDs, HDACi and MoAb will available for RRMM patients, ultimately increasing the survival expectancy of this particularly difficult group of patients. Whether a cure for MM patients will eventually be achieved is a burning and interesting question, that needs to be carefully addressed. Future and ongoing studies, also looking into the biology of MM and on the mechanism of resistance development, will help us answer this question and to balance risk and benefit in the treatment of myeloma. 


\section{Key issues}

- Despite these major advances, MM remains an incurable malignancy, with patients ultimately succumbing to their disease

- Outcomes of patients who have failed prior bortezomib- and lenalidomide-based therapies are particularly dismal

- Second generation proteasome inhibitors, third generation immunomodulatory drugs, histone deacetylase inhibitors and monoclonal antibodies have been developed and are under investigation, further increasing treatment options for RRMM patients

- Novel agent-based combinations are likely to increase treatment options for RRMM patients

- Triplet combinations demonstrated superior response rates and prolonged disease control when compared with two-drug regimens

- The addition of a third agent does not add any relevant additional toxicity.

- Salvage triplet therapies are likely to play a key role in overcoming drug-resistance and it is believed that they will further improve long-term outcomes of RRMM patients.

- A standard of care for the management of RRMM still cannot be defined.

- Optimal salvage therapy and treatment sequencing are still the matter of debate 


\section{Conflict of interests:}

Michele Cavo has received honoraria and has been a member of the advisory board for Celgene, Janssen and Millennium. The authors have no other relevant affiliations or financial involvement with any organization or entity with a financial interest in or financial conflict with the subject matter or materials discussed in the manuscript apart from those disclosed.

\section{References}

\section{Papers of interest- *}

\section{Papers of particular interest $-* *$}

1. Kumar SK, Rajkumar SV, Dispenzieri A, et al. Improved survival in multiple myeloma and the impact of novel therapies. Blood. 111(5), 2516-2520 (2008).*

A summary of the improved survival outcomes in MM patients with the use of novel therapies

2. Kumar SK, Lee JH, Lahuerta JJ, et al. Risk of progression and survival in multiple myeloma relapsing after therapy with IMiDs and bortezomib: A multicenter international myeloma working group study. Leukemia. 26(1), 149-157 (2012).

3. San Miguel J, Weisel K, Moreau P, et al. Pomalidomide plus low-dose dexamethasone versus high-dose dexamethasone alone for patients with relapsed and refractory multiple myeloma (MM-003): a randomised, open-label, phase 3 trial. Lancet Oncol. 14(11), 1055-1066 (2013).

Phase III trial demonstrating the effectiveness of Pomalidomide in patients relapsed or refractory to bortezomib and lenalidomide and leading to FDA and EMA approval for Pomalidomide 
4. Siegel DS, Martin T, Wang M, et al. A phase 2 study of single-agent carfilzomib (PX-171-003A1) in patients with relapsed and refractory multiple myeloma. Blood. 120(14), 2817-2825 (2012).*

Demonstration of the efficacy of Carflizomib in RRMM

5. Demo SD, Kirk CJ, Aujay MA, et al. Antitumor Activity of PR-171, a Novel Irreversible Inhibitor of the Proteasome. Cancer Res. 67(13), 6383-6391 (2007).

6. Chanan-Khan AA, Swaika A, Paulus A, et al. Pomalidomide: the new immunomodulatory agent for the treatment of multiple myeloma. Blood Cancer J. 3(9), e143 (2013).

7. Arastu-Kapur S, Anderl JL, Kraus M, et al. Nonproteasomal Targets of the Proteasome Inhibitors Bortezomib and Carfilzomib: a Link to Clinical Adverse Events. Clin. Cancer Res. 17(9), 2734-2743 (2011).

8. Lacy MQ, McCurdy AR. Pomalidomide. Blood. 122(14), 2305-2309 (2013).

9. Quach $H$, Ritchie $D$, Stewart AK, et al. Mechanism of action of immunomodulatory drugs (IMiDS) in multiple myeloma. Leukemia. 24(1), 22-32 (2009).

10. Parlati F, Lee SJ, Aujay M, et al. Carfilzomib can induce tumor cell death through selective inhibition of the chymotrypsin-like activity of the proteasome. Blood. 114(16), 3439-3447 (2009).

11. Lonial S, Vij R, Harousseau J-L, et al. Elotuzumab in Combination With Lenalidomide and LowDose Dexamethasone in Relapsed or Refractory Multiple Myeloma. J. Clin. Oncol. 30(16), 1953-1959 (2012).**

Phase III study demonstrating the effectivness of MoAb treatment in MM patients 
12. Jakubowiak AJ, Offidani M, Pegourie B, et al. A randomized phase II study of bortezomib $(B t z) /$ dexamethasone (dex) with or without elotuzumab (Elo) in patients (pts) with relapsed/refractory multiple myeloma (RRMM). J. Clin. Oncol. 33(Suppl), Abs 8573 (2015).

13. Lokhorst $\mathrm{H}$, Plesner $\mathrm{T}$, Gimsing $\mathrm{P}$, et al. Phase $\mathrm{I} / \mathrm{II}$ dose-escalation study of daratumumab in patients with relapsed or refractory multiple myeloma. J. Clin. Oncol. 31(Suppl), Abs 8512.

14. Plesner T, Arkenau T, Lokhorst H, et al. Preliminary Safety and Efficacy Data Of Daratumumab In Combination With Lenalidomide and Dexamethasone In Relapsed Or Refractory Multiple Myeloma. Blood. 122(21), 1986-1986 (2013).*

Demonstration of the efficacy of a antiCD38 antibody in RRMM patients

15. Dimopoulos M, Siegel DS, Lonial S, et al. Vorinostat or placebo in combination with bortezomib in patients with multiple myeloma (VANTAGE 088): a multicentre, randomised, double-blind study. Lancet Oncol. 14(11), 1129-1140 (2013).*

First demonstration in a Phase III trial of the efficacy of HDACi in MM patients

16. San-Miguel JF, Hungria VTM, Yoon S-S, et al. Panobinostat plus bortezomib and dexamethasone versus placebo plus bortezomib and dexamethasone in patients with relapsed or relapsed and refractory multiple myeloma: a multicentre, randomised, doubleblind phase 3 trial. Lancet Oncol. 15(11), 1195-1206 (2014).**

Phase III trial that lead to fast track approval by the FDA of Panobinostat in combination with bortezomib and dexamethasone for the treatment of RRMM.

17. Singhal S, Mehta J, Desikan R, et al. Antitumor Activity of Thalidomide in Refractory Multiple Myeloma. N. Engl. J. Med. 341(21), 1565-1571 (1999). 
18. Glasmacher A, Hahn C, Hoffmann F, et al. A systematic review of phase-II trials of thalidomide monotherapy in patients with relapsed or refractory multiple myeloma. Br. J. Haematol. 132(5), 584-593 (2006).

19. Von Lilienfeld-Toal M, Hahn-Ast C, Furkert K, et al. A systematic review of phase II trials of thalidomide/dexamethasone combination therapy in patients with relapsed or refractory multiple myeloma. Eur. J. Haematol. 81(4), 247-252 (2008).

20. Dimopoulos MA, Hamilos G, Zomas A, et al. Pulsed cyclophosphamide, thalidomide and dexamethasone: an oral regimen for previously treated patients with multiple myeloma. Hematol. J. Off. J. Eur. Haematol. Assoc. EHA. 5(2), 112-117 (2004).

21. García-Sanz R, González-Porras JR, Hernández JM, et al. The oral combination of thalidomide, cyclophosphamide and dexamethasone (ThaCyDex) is effective in relapsed/refractory multiple myeloma. Leukemia. 18(4), 856-863 (2004).

22. Offidani M, Bringhen S, Corvatta L, et al. Thalidomide-dexamethasone plus pegylated liposomal doxorubicin vs. thalidomide-dexamethasone: a case-matched study in advanced multiple myeloma. Eur. J. Haematol. 78(4), 297-302 (2007).

23. Garderet L, lacobelli S, Moreau $\mathrm{P}$, et al. Superiority of the Triple Combination of BortezomibThalidomide-Dexamethasone Over the Dual Combination of Thalidomide-Dexamethasone in Patients With Multiple Myeloma Progressing or Relapsing After Autologous Transplantation: The MMVAR/IFM 2005-04 Randomized Phase III Trial From the Chronic Leukemia Working Party of the European Group for Blood and Marrow Transplantation. J. Clin. Oncol. 30(20), 2475-2482 (2012). 
24. Richardson PG, Mitsiades C, Ghobrial I, Anderson K. Beyond single-agent bortezomib: combination regimens in relapsed multiple myeloma. Curr. Opin. Oncol. 18(6), 598-608 (2006).

25. Rosiñol L, Oriol A, Teruel Al, et al. Superiority of bortezomib, thalidomide, and dexamethasone (VTD) as induction pretransplantation therapy in multiple myeloma: a randomized phase 3 PETHEMA/GEM study. Blood. 120(8), 1589-1596 (2012).

26. Cavo M, Tacchetti P, Patriarca F, et al. Bortezomib with thalidomide plus dexamethasone compared with thalidomide plus dexamethasone as induction therapy before, and consolidation therapy after, double autologous stem-cell transplantation in newly diagnosed multiple myeloma: a randomised phase 3 study. The Lancet. 376(9758), 2075-2085 (2010).

27. Weber DM, Chen C, Niesvizky R, et al. Lenalidomide plus Dexamethasone for Relapsed Multiple Myeloma in North America. N. Engl. J. Med. 357(21), 2133-2142 (2007).

28. Dimopoulos M, Spencer A, Attal M, et al. Lenalidomide plus Dexamethasone for Relapsed or Refractory Multiple Myeloma. N. Engl. J. Med. 357(21), 2123-2132 (2007).

29. Rajkumar SV, Jacobus S, Callander NS, et al. Lenalidomide plus high-dose dexamethasone versus lenalidomide plus low-dose dexamethasone as initial therapy for newly diagnosed multiple myeloma: an open-label randomised controlled trial. Lancet Oncol. 11(1), 29-37 (2010).

30. Richardson PG, Siegel DS, Vij R, et al. Pomalidomide alone or in combination with low-dose dexamethasone in relapsed and refractory multiple myeloma: a randomized phase 2 study. Blood. 123(12), 1826-1832 (2014). 
31. Leleu X, Attal M, Arnulf B, et al. Pomalidomide plus low-dose dexamethasone is active and well tolerated in bortezomib and lenalidomide-refractory multiple myeloma: Intergroupe Francophone du Myélome 2009-02. Blood. 121(11), 1968-1975 (2013).

32. Larocca A, Montefusco V, Bringhen S, et al. Pomalidomide, cyclophosphamide, and prednisone for relapsed/refractory multiple myeloma: a multicenter phase $1 / 2$ open-label study. Blood. 122(16), 2799-2806 (2013).

33. Baz R, Martin TG, Alsina M, et al. Pomalidomide, Cyclophosphamide, and Dexamethasone Is Superior to Pomalidomide and Dexamethasone in Relapsed and Refractory Myeloma: Results of a Multicenter Randomized Phase II Study. Blood. 124(21), 303-303 (2014).

34. Lacy MQ, LaPlant BR, Laumann KM, et al. Pomalidomide, Bortezomib and Dexamethasone (PVD) for Patients with Relapsed Lenalidomide Refractory Multiple Myeloma (MM). Blood. 124(21), 304-304 (2014).

35. Shah JJ, Stadtmauer EA, Abonour R, et al. Phase I/II Dose Expansion Of a Multi-Center Trial Of Carfilzomib and Pomalidomide With Dexamethasone (Car-Pom-d) In Patients With Relapsed/Refractory Multiple Myeloma. Blood. 122(21), 690-690 (2013).

36. Shah JJ, Stadtmauer EA, Abonour R, et al. Carfilzomib, pomalidomide, and dexamethasone (CPD) in patients with relapsed and/or refractory multiple myeloma. Blood. , blood-201505-643320 (2015).

37. Richardson PG, Sonneveld P, Schuster MW, et al. Bortezomib or High-Dose Dexamethasone for Relapsed Multiple Myeloma. N. Engl. J. Med. 352(24), 2487-2498 (2005). 
38. Richardson PG, Sonneveld P, Schuster M, et al. Extended follow-up of a phase 3 trial in relapsed multiple myeloma: final time-to-event results of the APEX trial. Blood. 110(10), 3557-3560 (2007).

39. Jagannath S, Richardson PG, Barlogie B, et al. Bortezomib in combination with dexamethasone for the treatment of patients with relapsed and/or refractory multiple myeloma with less than optimal response to bortezomib alone. Haematologica. 91(7), 929-934 (2006).

40. Mitsiades N, Mitsiades CS, Richardson PG, et al. The proteasome inhibitor PS-341 potentiates sensitivity of multiple myeloma cells to conventional chemotherapeutic agents: therapeutic applications. Blood. 101(6), 2377-2380 (2003).

41. Orlowski RZ, Nagler A, Sonneveld P, et al. Randomized Phase III Study of Pegylated Liposomal Doxorubicin Plus Bortezomib Compared With Bortezomib Alone in Relapsed or Refractory Multiple Myeloma: Combination Therapy Improves Time to Progression. J. Clin. Oncol. 25(25), 3892-3901 (2007).

42. Gozzetti A, Fabbri A, Oliva S, et al. Weekly Bortezomib, Pegylated Liposomal Doxorubicin, and Dexamethasone Is a Safe and Effective Therapy for Elderly Patients With Relapsed/Refractory Multiple Myeloma. Clin. Lymphoma Myeloma Leuk. 10(1), 68-72 (2010).

43. Palumbo A, Gay F, Bringhen S, et al. Bortezomib, doxorubicin and dexamethasone in advanced multiple myeloma. Ann. Oncol. 19(6), 1160-1165 (2008).

44. Waterman GN, Yellin O, Swift RA, et al. A modified regimen of pegylated liposomal doxorubicin, bortezomib, and dexamethasone is effective and well tolerated in the treatment of relapsed or refractory multiple myeloma. Ann. Hematol. 90(2), 193-200 (2010). 
45. Richardson PG, Xie W, Jagannath S, et al. A phase 2 trial of lenalidomide, bortezomib, and dexamethasone in patients with relapsed and relapsed/refractory myeloma. Blood. 123(10), 1461-1469 (2014).

46. Offidani M, Corvatta L, Maracci L, et al. Efficacy and tolerability of bendamustine, bortezomib and dexamethasone in patients with relapsed-refractory multiple myeloma: a phase II study. Blood Cancer J. 3(11), e162 (2013).

47. Ludwig $\mathrm{H}$, Kasparu $\mathrm{H}$, Leitgeb $\mathrm{C}$, et al. Bendamustine-bortezomib-dexamethasone is an active and well-tolerated regimen in patients with relapsed or refractory multiple myeloma. Blood. 123(7), 985-991 (2014).

48. Rodon P, Hulin C, Pegourie B, et al. Phase II study of bendamustine, bortezomib and dexamethasone as second-line treatment for elderly patients with multiple myeloma: the Intergroupe Francophone du Myelome 2009-01 trial. Haematologica. 100(2), e56-e59 (2015).

49. Stewart AK, Rajkumar SV, Dimopoulos MA, et al. Carfilzomib, Lenalidomide, and Dexamethasone for Relapsed Multiple Myeloma. N. Engl. J. Med. 372(2), 142-152 (2015).**

Phase III trial leading to FDA approval of Carfilzomib in combination with lenalidomide and dexamethasone for the treatment of patients with relapsed MM who have received 1 to 3 prior lines of therapy.

50. Kumar SK, Bensinger WI, Zimmerman TM, et al. Phase 1 study of weekly dosing with the investigational oral proteasome inhibitor ixazomib in relapsed/refractory multiple myeloma. Blood. 124(7), 1047-1055 (2014). 
51. Maloney DG, Liles TM, Czerwinski DK, et al. Phase I clinical trial using escalating single-dose infusion of chimeric anti-CD20 monoclonal antibody (IDEC-C2B8) in patients with recurrent Bcell lymphoma. Blood. 84(8), 2457-2466 (1994).

52. Teo EC, Chew Y, Phipps C. A review of monoclonal antibody therapies in lymphoma. Crit. Rev. Oncol. Hematol. , Epub ahead of print (2015).

53. Lonial S, Dimopoulos M, Palumbo A, et al. Elotuzumab Therapy for Relapsed or Refractory Multiple Myeloma. N. Engl. J. Med. 373(7), 621-631 (2015).

54. Jakubowiak AJ, Benson DM, Bensinger W, et al. Phase I Trial of Anti-CS1 Monoclonal Antibody Elotuzumab in Combination With Bortezomib in the Treatment of Relapsed/Refractory Multiple Myeloma. J. Clin. Oncol. 30(16), 1960-1965 (2012).

55. Lokhorst HM, Plesner T, Laubach JP, et al. Targeting CD38 with Daratumumab Monotherapy in Multiple Myeloma. N. Engl. J. Med. 373(13), 1207-1219 (2015).

56. Plesner T, Arkenau H-T, Lokhorst HM, et al. Safety and efficacy of daratumumab with lenalidomide and dexamethasone in relapsed or relapsed, refractory multiple myeloma. ASCO Meet. Abstr. 32(15_suppl), 8533 (2014).

57. Plesner T, Arkenau H-T, Lokhorst HM, et al. Safety and Efficacy of Daratumumab with Lenalidomide and Dexamethasone in Relapsed or Relapsed, Refractory Multiple Myeloma. Blood. 124(21), 84-84 (2014).

58. Moreau P, Mateos M-V, Bladé J, et al. An Open-Label, Multicenter, Phase 1b Study of Daratumumab in Combination with Backbone Regimens in Patients with Multiple Myeloma. Blood. 124(21), 176-176 (2014). 
Table 1: Principal trials comparing two- versus three-drug combinations in RRMM

\begin{tabular}{|c|c|c|c|c|c|c|c|c|c|c|c|}
\hline Author & Study & Pts & Drugs & ORR ( $\geq$ PR) & $P$ value & $\mathrm{nCR} / \mathrm{CR}$ & $P$ value & PFS (mo) & $P$ value & os & $P$ value \\
\hline $\begin{array}{l}\text { Offidani } \\
(2008)[46]\end{array}$ & $\begin{array}{l}\text { Retrospective, } \\
\text { case match }\end{array}$ & $\begin{array}{l}47 \\
47\end{array}$ & $\begin{array}{l}\text { Thal-dex } \\
\text { ThaDD }\end{array}$ & $\begin{array}{l}59.5 \% \\
75.5 \%\end{array}$ & 0.07 & $\begin{array}{l}10.5 \% \\
30 \%\end{array}$ & 0.002 & $\begin{array}{l}11.5 \\
21\end{array}$ & 0.009 & $\begin{array}{l}20 \mathrm{mo} \\
35.5 \mathrm{mo}\end{array}$ & 0.01 \\
\hline $\begin{array}{l}\text { Garderet } \\
(2012)[23]\end{array}$ & $\begin{array}{l}\text { Randomized } \\
\text { Phase III }\end{array}$ & $\begin{array}{l}134 \\
135\end{array}$ & $\begin{array}{l}\text { TD } \\
\text { VTD }\end{array}$ & $\begin{array}{l}72 \% \\
87 \%\end{array}$ & 0.003 & $\begin{array}{l}21 \% \\
45 \%\end{array}$ & 0.001 & $\begin{array}{l}13.6 \\
18.3\end{array}$ & 0.001 & $\begin{array}{l}\text { 65\% @ 2-y } \\
71 \% @ 2-y\end{array}$ & 0.09 \\
\hline $\begin{array}{l}\text { Stewart } \\
(2015)[49]\end{array}$ & $\begin{array}{l}\text { Randomized } \\
\text { Phase III }\end{array}$ & $\begin{array}{l}396 \\
396\end{array}$ & $\begin{array}{l}\text { Rd } \\
\text { Rd-Carfilzomib }\end{array}$ & $\begin{array}{l}66.7 \% \\
87.1 \%\end{array}$ & $<0.001$ & $\begin{array}{l}9.3 \% * \\
31.8 \%\end{array}$ & $<0.001$ & $\begin{array}{l}17.6 \\
26.3\end{array}$ & $<0.001$ & $\begin{array}{l}65.0 \% @ 2-y \\
73.3 \% @ 2-y\end{array}$ & / \\
\hline $\begin{array}{l}\text { Lonial } \\
(2015)[53]\end{array}$ & $\begin{array}{l}\text { Randomized } \\
\text { Phase III }\end{array}$ & $\begin{array}{l}325 \\
321\end{array}$ & $\begin{array}{l}\text { Rd } \\
\text { Rd-Elotuzumab }\end{array}$ & $\begin{array}{l}66 \% \\
79 \%\end{array}$ & $<0.001$ & $\begin{array}{l}11 \% * \\
11 \%\end{array}$ & I & $\begin{array}{l}14.9 \\
19.4\end{array}$ & $<0.001$ & $N A$ & I \\
\hline $\begin{array}{l}\text { San Miguel } \\
(2014)[16]\end{array}$ & $\begin{array}{l}\text { Randomized } \\
\text { Phase III }\end{array}$ & $\begin{array}{l}381 \\
387\end{array}$ & $\begin{array}{l}\text { VD-placebo } \\
\text { VD-Panobinostat }\end{array}$ & $\begin{array}{l}54.6 \% \\
60.7 \%\end{array}$ & 0.09 & $\begin{array}{l}15.7 \% \\
27.6 \%\end{array}$ & $<0.001$ & $\begin{array}{l}8.1 \\
12\end{array}$ & $<0.001$ & $\begin{array}{l}30.4 \\
33.6\end{array}$ & 0.26 \\
\hline $\begin{array}{l}\text { Jakubowiak } \\
\text { (2015) [12] }\end{array}$ & $\begin{array}{l}\text { Randomized } \\
\text { Phase II }\end{array}$ & $\begin{array}{l}75 \\
77\end{array}$ & $\begin{array}{l}\text { VD } \\
\text { VD-Elotuzumab }\end{array}$ & $\begin{array}{l}63 \% \\
66 \%\end{array}$ & NA & NA & / & $\begin{array}{l}6.9 \\
9.7\end{array}$ & 0.08 & NA & / \\
\hline
\end{tabular}

Abbreviations: ORR: overall response rate; PR: partial response; $n C R$ : near complete response; CR: complete response; PFS: progression free survival; OS overall survival; ThaDD: thalidomide-

dexamethasone-doxorubicin; TD: thalidomide-dexamethasone; VTD: bortezomib-thalidomide-dexamethasone; Rd: lenalidomide- low dose dexamethasone; VD: bortezomib-dexamethasone; NA: not available.

* only $\geq \mathrm{CR}$ 
Table 2: Principal grade III-IV toxicities of three-drug combination regimen in RRMM

\begin{tabular}{|c|c|c|c|c|c|c|c|c|c|c|}
\hline Author & Treatment & Pts & $\begin{array}{l}\text { Anemi } \\
\text { a }\end{array}$ & $\begin{array}{l}\text { Neutropeni } \\
\text { a }\end{array}$ & $\begin{array}{l}\text { Thrombocytopeni } \\
\text { a }\end{array}$ & $\begin{array}{l}\text { Infection } \\
\text { s }\end{array}$ & PNP & $\begin{array}{l}\text { Cardia } \\
\text { c } \\
\text { events }\end{array}$ & TE & $\begin{array}{l}\text { GI } \\
\text { event } \\
\text { s }\end{array}$ \\
\hline $\begin{array}{l}\text { Offidani } \\
\text { (2008)[46] }\end{array}$ & ThaDD & 47 & NR & $25 \%$ & $3 \mathrm{pts}$ & $23 \%$ & $2 \%$ & 0 & $\begin{array}{l}6 \\
\mathrm{pt} \\
\mathrm{s}\end{array}$ & NR \\
\hline $\begin{array}{l}\text { Garderet } \\
(2012)[23]\end{array}$ & VTD & $\begin{array}{l}13 \\
3\end{array}$ & $8 \%$ & $11 \%$ & $17 \%$ & $14 \%$ & $31 \%$ & $2 \%$ & $6 \%$ & $8 \%$ \\
\hline $\begin{array}{l}\text { Stewart } \\
(2015)[49]\end{array}$ & $\begin{array}{l}\text { Rd- } \\
\text { Carfilzomib }\end{array}$ & $\begin{array}{l}39 \\
2\end{array}$ & $17.9 \%$ & $29.6 \%$ & $16.6 \%$ & $1.8 \%$ & $\begin{array}{l}17 \% \\
\text { (all } \\
\text { grades } \\
\text { ) }\end{array}$ & 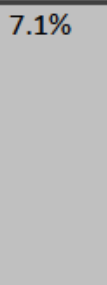 & NR & $5.6 \%$ \\
\hline $\begin{array}{l}\text { Lonial } \\
\text { (2015)[53] }\end{array}$ & $\begin{array}{l}\text { Rd- } \\
\text { Elotuzumab }\end{array}$ & $\begin{array}{l}32 \\
1\end{array}$ & $19 \%$ & $34 \%$ & $19 \%$ & NR & NR & NR & NR & $6 \%$ \\
\hline $\begin{array}{l}\text { San Miguel } \\
\text { (2014)[16] }\end{array}$ & $\begin{array}{l}\text { VD- } \\
\text { Panobinosta } \\
\text { t }\end{array}$ & $\begin{array}{l}38 \\
7\end{array}$ & $18 \%$ & $35 \%$ & $68 \%$ & $13 \%$ & $17 \%$ & NR & NR & $46 \%$ \\
\hline $\begin{array}{l}\text { Jakubowia } \\
\text { k } \\
\text { (2015) [12] }\end{array}$ & $\begin{array}{l}\text { VD- } \\
\text { Elotuzumab }\end{array}$ & 77 & NR & NR & $9 \%$ & $19 \%$ & NR & NR & NR & NR \\
\hline
\end{tabular}

Abbreviations: PNP: peripheral neuropathy; TE: thromboembolic events; GI: gastrointestinal events, including diarrhoea, constipation, vomiting, nausea; NR: not reported 